\title{
Pancreatic Adenosquamous Carcinoma
}

National Cancer Institute

\section{Source}

National Cancer Institute. Pancreatic Adenosquamous Carcinoma. NCI Thesaurus. Code C5721.

A carcinoma that arises from the pancreas showing both ductal and squamous differentiation. The squamous component should represent at least $30 \%$ of the malignant cellular infiltrate. The prognosis is usually worse than that of ductal adenocarcinoma. 